\title{
AVALIAÇÃO DE DEPRESSÃO, ANSIEDADE E NÍVEL COGNITIVO EM IDOSOS DE UMA INSTITUIÇÃO NO MUNICÍPIO DE CRICIÚMA, SANTA CATARINA
}

\begin{abstract}
Evaluation of depression, anxiety and cognitive level in elderly from an institution in Criciúma city, state of Santa Catarina
\end{abstract}

\begin{abstract}
Aline Knevitz Lippert ${ }^{1}$, Fernanda de Souza Fernandes ${ }^{2}$, Rosa Nadir Teixeira Jerônimo ${ }^{3}$, Karin Martins Gomes ${ }^{4}$
\end{abstract}

\footnotetext{
${ }^{1}$ Acadêmica do último ano do Curso de Psicologia da Universidade do Extremo Sul Catarinense, Criciúma, SC.

${ }^{2}$ Professora Especialista e Mestranda do Curso de Psicologia da Universidade do Extremo Sul Catarinense, Criciúma, SC.

${ }^{3}$ Professora Mestre do Curso de Psicologia da Universidade do Extremo Sul Catarinense, Criciúma, SC.

${ }^{4}$ Professora Doutora do Curso de Psicologia da Universidade do Extremo Sul Catarinense, Criciúma, SC.
}

\section{Endereço para correspondência:}

*Karina Martina Gomes

Av. Universitária, 1105 - Bairro Universitário

88806-00, Criciúma - SC

Email:karin@unesc.net 


\section{RESUMO}

A pessoa idosa que está institucionalizada pode apresentar um declínio maior principalmente em relação aos aspectos emocionais pelo ambiente em que se encontra. Esta é uma pesquisa de caráter quantitativo, descritiva e observacional com amostra não probabilística e intencional. Participaram 15 idosos institucionalizados, com idades entre 60 a 80 anos. O presente estudo teve como objetivo avaliar indícios de depressão, ansiedade e quociente intelectual. Os instrumentos utilizados foram o WASI, o inventário BAI e escala de depressão geriátrica - GDS. Os resultados demonstraram que 09 idosos apresentaram QI extremamente abaixo da média, 07 idosos apresentaram depressão e 04 idosos indicaram ansiedade. Entre os que apresentaram ansiedade, 02 deles também apresentaram depressão. Entre esses participantes, a maioria possui escolaridade baixa, 09 não praticam exercícios físicos e nenhum participa de outras atividades além da instituição. Diante dos resultados sugere-se ter relação a comorbidade entre depressão e ansiedade, além de baixa escolaridade.

Palavras - chave: Idosos; Depressão; Ansiedade.

\section{ABSTRACT}

The institutionalized elderly person can present a major decline mainly on emotional aspects due to the environment where the person lives. This research is quantitative, descriptive and observational with non-probabilistic and intentional sampling. The population is characterized by 15 institutionalized elderly people, with age between 60 to 80 years old. The present study aimed to evaluate indications of depression, anxiety and intellectual quotient. The utilized instruments were WASI, BAI inventory and geriatric depression scale - GDS. The results showed that 09 elderly people presented extremely below average IQ, 07 presented depression and 04 indicated anxiety. Among the ones who presented anxiety, 02 of them also presented depression. Among these participants, most of them have low educational level, 09 do not practice physical exercises e none of them do any other activity beyond the institution. It is suggested that there is a relation between comorbidity between 
Artigo Original

Atenção à Saúde

depression and anxiety, in addition to low schooling.

Keywords: Elderly; Depression; Anxiety.

\section{INTRODUÇÃO}

O envelhecimento humano representa um processo biopsicossocial e, por essa natureza, provoca demandas complexas e exige cuidado diferenciado ${ }^{1}$. Com o passar dos anos, o aumento da prevalência de problemas específicos do envelhecimento como diminuição da acuidade visual e auditiva, incontinências, incidências de quedas, demência, depressão e isolamento fazem parte do marco dos 65 anos $^{2}$.

Segundo o Ministério da Saúde, a longevidade é uma conquista. No entanto, há importantes diferenças entre os países desenvolvidos e os países em desenvolvimento. Enquanto, nos primeiros, o envelhecimento ocorreu associado às melhorias nas condições gerais de vida, nos outros, esse processo acontece de forma rápida, sem tempo para uma reorganização social e da área de saúde adequada para atender às novas demandas emergentes ${ }^{3}$.

Para o ano de 2050, a expectativa no Brasil, assim como em todo o mundo, é de que existirão mais idosos que crianças abaixo de 15 anos; um fenômeno nunca observado antes ${ }^{3}$. Esses dados demonstram que as pessoas têm vivido mais, porém, há agravos na qualidade de vida, tendo em vista o significativo aumento das doenças crônicas que acometem as pessoas, especialmente, durante o processo de envelhecimento ${ }^{2}$.

Envelhecer com uma má qualidade de vida é um problema, pois não adiantará de nada o aumento grandioso do número de idosos se não houver recursos necessários que supram os cuidados com esta população. 0 envelhecimento humano faz parte do processo biopsicossocial e, por isso, há busca por um cuidado diferenciado ${ }^{4}$.

Com o aumento da população de idosos, houve também um aumento no número de doenças, o que pode ser comum nessa idade. Estudos mostram que um dos diagnósticos mais encontrados nessa população é o transtorno de humor 
Artigo Original

Atenção à Saúde

acompanhado de ansiedade (15,4\% dos casos), que se caracteriza por um sentimento vago e desagradável de medo e apreensão ${ }^{4}$.

É possível a ocorrência de algum transtorno de humor no decorrer do envelhecimento, assim como há hipótese de que a pessoa idosa também pode desenvolver um comprometimento cognitivo, já que o processo do envelhecimento acarreta várias limitações, tanto físicas, como emocionais e sociais, podendo estes estar associados aos transtornos de humor e declínio cognitivo. Sendo assim o objetivo desta pesquisa foi avaliar a depressão, ansiedade e nível cognitivo em idosos institucionalizados.

\section{MÉTODO}

\section{Amostra}

Esta é uma pesquisa de caráter quantitativo, descritiva e observacional. Além de ser um estudo de campo, utilizando-se de uma amostra não probabilística e intencional ${ }^{5}$. Os critérios de inclusão utilizados foram: idosos entre 60 a 80 anos de idade, sem Transtorno Mental diagnosticado ou doenças como qualquer tipo de demência, diabetes, deficiências intelectuais ou limitação de aprendizado, doenças neurodegenerativas, acidente vascular, nefropatias, com retinopatias avançada ou que fazem uso de medicamentos psicotrópicos.

Os idosos avaliados estão em uma instituição asilar da Cidade de Criciúma, Santa Catarina, e concordaram em participar da pesquisa mediante assinatura do Termo de Consentimento Livre e Esclarecido (TCLE). O presente estudo foi devidamente aprovado pelo Comitê de ética em Pesquisa com Seres Humanos da UNESC, tendo como base a Resolução 466/12, parecer 1.869.914.

\section{Procedimento}

Primeiramente foram coletadas as assinaturas do TCLE, em seguida, foi realizado um questionário sócio demográfico para levantar características dos indivíduos (como nome, idade, sexo, escolaridade, estado civil, atividades físicas, sociais, tempo de permanência no asilo e composição familiar). Posteriormente, foi 
aplicado a Escala Wechsler Abreviada de Inteligência (WASI) ${ }^{6}$ para avaliar nível cognitivo, assim como o Inventário Beck de Ansiedade (BAI) ${ }^{7}$ que faz parte das Escalas Beck, e a Escala de Depressão Geriátrica (GDS) ${ }^{8}$, para avaliar ansiedade e depressão respectivamente.

A escala WASI é um instrumento de avaliação da inteligência, aplicável a crianças de 06 anos a idosos de 89 anos de idade. Fornece informações sobre os Qls Total, de Execução e Verbal a partir de quatro subtestes (Vocabulário, Cubos, Semelhanças e Raciocínio Matricial), em um curto espaço de tempo. A escala ainda fornece a possibilidade de avaliação do QI Total com apenas dois subtestes (Vocabulário e Raciocínio Matricial) ${ }^{6}$.

O BAl tem por objetivo mensurar a intensidade da ansiedade, apresentando como resultado grau mínimo de ansiedade, leve, moderado ou grave. E o GDS constitui em um instrumento mais empregado para avaliar sintomas depressivos em populações geriátricas. Este instrumento possui 15 perguntas, o qual o resultado de 5 ou mais pontos dá indício de sintomas depressivos, sendo que o escore igual ou maior que 11 caracteriza depressão grave ${ }^{7}$.

Os dados foram transferidos para um banco de dados em planilhas do software Microsoft Excel, sendo expressos em frequência e percentual.

\section{ANÁLISE E DISCUSSÃO DOS RESULTADOS}

Os dados apresentados a seguir estão relacionados primeiramente aos resultados encontrados no questionário sociodemográfico. Neste estudo a proporção foi maior do sexo masculino, sendo 09 homens e 06 mulheres entre os 15 idosos pesquisados.

Quanto ao estado civil, 07 idosos institucionalizados são viúvos/viúvas, 03 são divorciados, 04 são casados e 01 é solteiro. Entre eles, 12 idosos possuem mais de 02 filhos e somente 02 idosos não tem filhos. Apresentando também em sua maioria (12) escolaridade baixa, ou seja, ensino fundamental incompleto. Somente um participante possui ensino médio completo, outro (01) possui ensino médio incompleto e um possui ensino superior incompleto.

Dos idosos pesquisados, 09 não realizam atividades físicas e nenhum participa de outras atividades fora da instituição asilar e 06 idosos realizam 
Artigo Original

Atenção à Saúde

atividades físicas. Entre os idosos pesquisados, 05 estão institucionalizados há mais de 07 anos, outros 05 idosos estão no asilo há mais de 02 anos, 03 deles está há mais de 02 meses e 02 deles está há apenas 01 mês.

$\mathrm{Na}$ avaliação de depressão realizada através do instrumento GDS, foi constatado que entre os 15 participantes, 07 idosos entre 60 e 80 anos apresentaram indícios de depressão. O resultado pode ser observado através do quadro 1.

\begin{tabular}{|c|c|}
\hline Número de Participantes & Resultado da Escala GDS \\
\hline 2 & 5 \\
\hline 1 & 6 \\
\hline 1 & 7 \\
\hline 1 & 8 \\
\hline 2 & 9 \\
\hline
\end{tabular}

Quadro 1: Resultado da Escala de Depressão GDS. 5 pontos ou mais indica indício de depressão e 11 pontos ou mais indica depressão grave ${ }^{7}$.

Fonte: Dados da Pesquisa.

Dos participantes que apresentaram depressão, 04 entre estes são mulheres e 03 homens. Pode-se observar que o resultado variou de 5 pontos até 9 pontos o qual indica indício de depressão, nenhum obteve resultado de depressão grave.

Fazendo a relação entre o resultado de depressão e atividade física, foi observado que somente 01 (mulher) dos idosos que apresentaram depressão realiza atividades físicas; mas os outros 06 idosos com indício de depressão, não fazem atividades físicas.

Em relação à depressão, na pesquisa de Cheik e colaboradores foi realizada uma comparação de níveis de depressão em idosos sedentários e idosos praticantes de alguma atividade física. Como resultado foi observado, que os idosos que já praticavam inicialmente alguma atividade como caminhada tiveram uma redução satisfatória nos níveis de depressão, já os idosos que não praticavam atividade física mantiveram seus índices de ansiedade e depressão ${ }^{9}$.

A depressão é o transtorno psiquiátrico mais comum entre os idosos, 
podendo afetar a qualidade de vida, aumentando a carga econômica por seus custos diretos e indiretos e, pode levar a tendências suicidas. Pessoas deprimidas mostram-se insatisfeitas com o que Ihes é oferecido, havendo interrupção em seus estilos de vida, redução de seu nível socioeconômico quando ficam impossibilitados de trabalharem ${ }^{10}$.

Entre todas as fases do desenvolvimento humano, a depressão pode estar presente também na fase do envelhecimento, pois é um período da vida em que muitas funções do corpo humano já estão limitadas. Além da diminuição das relações sociais, a saúde física e emocional do idoso também pode estar comprometida, e o isolamento pode ser um fator que desencadeia a depressão. Esta pesquisa demonstrou que dos 15 idosos pesquisados, 07 entre eles apresentaram indícios de depressão e, somente 01 deles pratica atividades físicas; mas nenhum entre eles participam de atividades sociais além da instituição asilar.

A ocorrência de depressão em idosos que permanecem em instituições asilares pode estar relacionado com o afastamento do convívio social, pois devido ao fato de permanecerem institucionalizados, pode não existir motivos que leve os idosos a se relacionarem com outras pessoas ${ }^{9}$.

O índice de ocorrência de qualquer transtorno de humor é de 4,9\%, sendo que a depressão é a mais prevalente, com taxas de 4,0\% a 9,7\% entre idosos com 55 a 85 anos de idade. $\mathrm{E}$ a depressão está ainda associada com a incapacidade funcional, comorbidades médicas e privação social ${ }^{11}$.

Entre os transtornos psiquiátricos, a depressão é a que mais ocorre entre os idosos. A ocorrência do quadro de depressão associado ao desenvolvimento da Doença de Alzheimer (DA) é comum, mas possivelmente o surgimento da depressão inicia antes do desenvolvimento da Doença de Alzheimer. Nesse caso, a depressão pode ser classificada como um dos sintomas iniciais da DA, a qual pode surgir muitos anos antes do comprometimento cognitivo do indivíduo ${ }^{12}$.

Em relação ao diagnóstico diferencial entre demência e transtornos psiquiátricos, a depressão é um dos principais diagnósticos diferenciais de demência. Mas é necessário lembrar que depressão e demência podem ser doenças simultâneas, e que depressão, acontecendo antes de um quadro demencial, pode representar um fator de risco ou precedente de demência ${ }^{12}$.

Não apenas a depressão pode surgir na fase da terceira idade, mas a 
Artigo Original

Atenção à Saúde

ansiedade pode ser encontrada ${ }^{4}$. Na avaliação do nível de ansiedade, realizada pelo instrumento BAl, pode-se destacar que, entre os 15 idosos entrevistados, 03 apresentaram nível leve de ansiedade e 01 apresentou nível moderado, estes estão entre 65 e 80 anos de idade, conforme a figura 1.

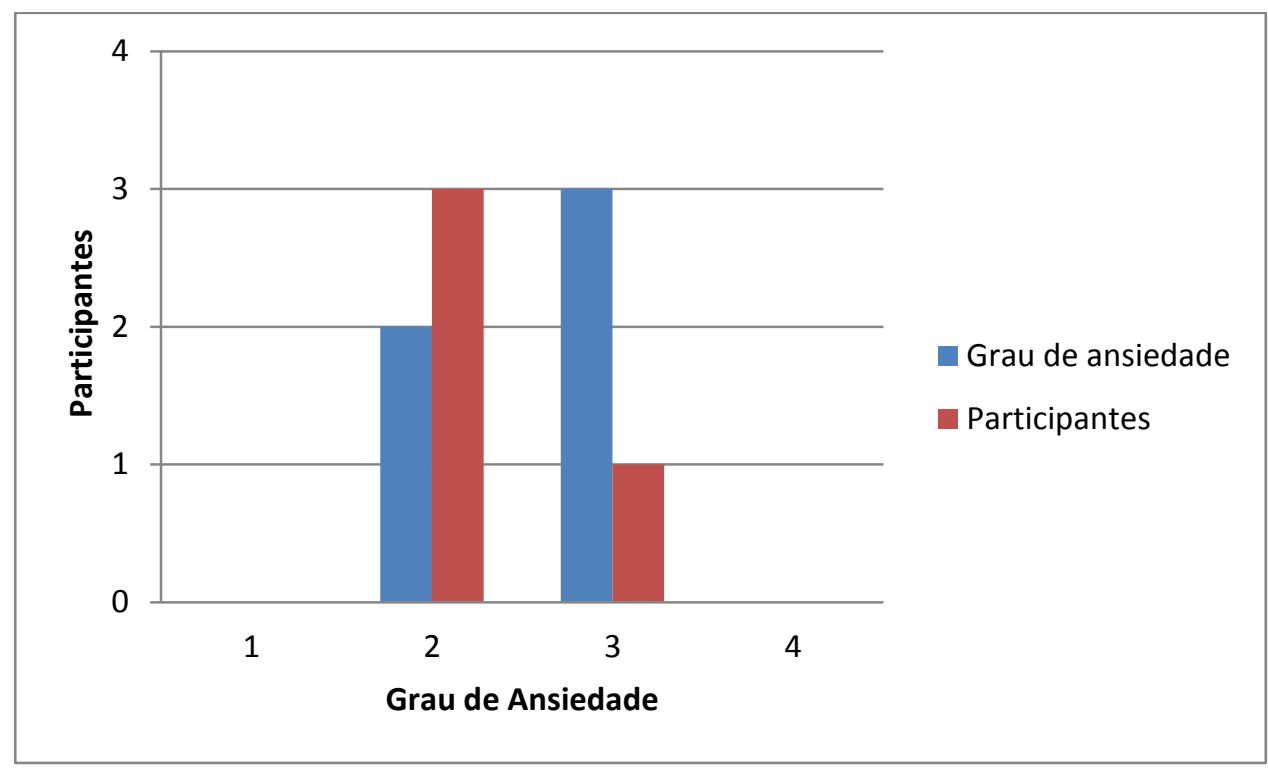

Figura 1: Ansiedade em idosos institucionalizados.

Escala de Ansiedade (BAI). Onde nos números 1 refere-se ao nível mínimo de ansiedade, 2 ao leve, 3 moderado, 4 grave.

Fonte: Dados da Pesquisa.

Dos 04 idosos que indicaram ansiedade, 03 deles apresentaram nível leve de ansiedade e 01 idoso apresentou nível moderado de ansiedade. Entre esses 04 que apresentaram ansiedade, 03 são homens. Um idoso que foi avaliado com nível moderado de ansiedade também apresentou indício de depressão, e um idoso que indicou ansiedade leve também apresentou indícios de depressão.

Nesta pesquisa, é importante ressaltar que 02 idosos que apresentaram depressão e ansiedade não participam de nenhuma atividade social fora da instituição asilar, não praticam exercícios físicos e possuem escolaridade baixa. A ansiedade e os transtornos depressivos são alterações que acontecem com muita frequência entre os idosos, sendo que são as maiores causas apontadas como sofrimento emocional e diminuição da qualidade de vida. Podendo também afetar o sistema de saúde público, devido à sua alta morbidade e mortalidade ${ }^{13}$. 
Dessa forma, verifica-se que tanto a depressão como a ansiedade, podem causar problemas na vida dos idosos, afetando assim, o desempenho físico, emocional e até mesmo social, pois o idoso que possui depressão ou indícios de ansiedade pode querer se isolar e isso causará prejuízos no próprio indivíduo e no relacionamento com os outros ${ }^{9}$.

Na avaliação de nível cognitivo (Quociente Intelectual - QI), os resultados da aplicação do Teste WASI estão apresentados na figura 2.

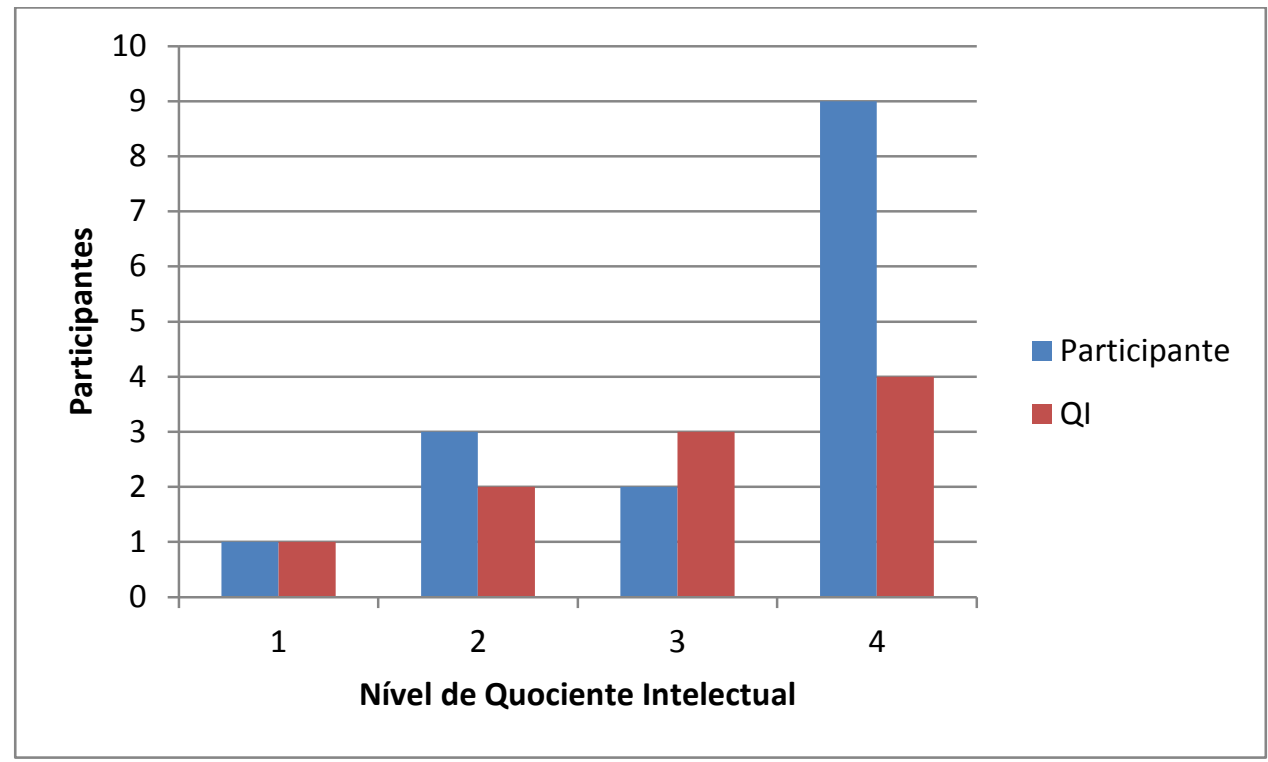

Figura 2: Nível cognitivo em idosos institucionalizados.

Resultado do Teste WASI (QI's). Onde nos números 1 refere-se na média para sua idade, 2 média inferior para sua idade, 3 limítrofe e 4 extremamente abaixo da média para sua idade ${ }^{6}$.

Fonte: Dados da Pesquisa.

Pode-se observar que 09 idosos, entre 65 e 80 anos, apresentaram QI extremamente abaixo da média, 02 idosos entre 62 e 80 anos, apresentaram QI limítrofe, 03 idosos entre 63 e 80 anos, apresentaram QI na média inferior e somente 01 idoso com 60 anos, apresentou QI na média.

Podemos relacionar estes dados com a escolaridade, 12 idosos, entre 62 e 80 anos, dos 15 entrevistados, possuem baixa escolaridade, ou seja, possuem ensino fundamental incompleto e este fator pode ter relação com o baixo nível cognitivo que apresentaram.

É importante ressaltar que o desenvolvimento do conhecimento em 


\section{Atenção à Saúde}

relação às estruturas fonológicas e gramaticais da língua com o objetivo de aprender a leitura e a escrita, resulta em mudanças arquiteturais e funcionais no cérebro. $\mathrm{O}$ que pode influenciar não somente na habilidade linguística de uma pessoa, mas também na sua maneira de processar e representar informações não linguísticas, como a habilidade de processar informações espaciais, de nomear objetos em duas dimensões, a memória de trabalho, o pensamento lógico e abstrato. Além disso, pessoas analfabetas e que possuem escolaridade muito baixa formam um grupo no qual o índice cognitivo é mais comprometido em relação à indivíduos com maior grau educacional ${ }^{14}$.

O comprometimento cognitivo afeta a capacidade funcional do indivíduo no seu dia a dia, implicando na perda de independência e autonomia, a qual varia de acordo com o grau de gravidade, com consequente perda da qualidade de vida do idoso. Por outro lado, a noção de autonomia, no que se refere à interdependência desta com uma memória íntegra, relaciona-se à capacidade individual de cuidar de si mesmo, executar tarefas que the permitam a adaptação psicossocial e ser responsável também pelos próprios atos ${ }^{15}$.

Lacerda e colaboradores ainda afirmam que o grau de dependência pode variar de acordo com a instituição acolhedora. Isso acontece, porque a instituição passa a assumir todas as responsabilidades que originalmente seria do idoso levando a um aumento de sua dependência e consequente a diminuição da capacidade funcional ${ }^{9}$.

Idosos que estão institucionalizados apresentam um perfil diferenciado, pois necessitam de atenção, de suporte e serviços especializados, já que a grande maioria é fragilizada, pode assim, apresentar morbidades físicas ou mentais, o que leva a uma tendência de prejuízos à saúde ${ }^{16}$.

Algumas situações são também marcadas pelo conflito familiar e resultam na procura, pela família ou, às vezes, até pelo próprio idoso pela instituição asilar. Em contrapartida, muitas famílias não conseguem manter o idoso dependente em casa porque o cuidado pode se tornar difícil e desgastante, tanto fisicamente, quanto emocionalmente ${ }^{17}$.

Pode-se afirmar que manter a capacidade funcional tornou-se o novo paradigma para o idoso. A perda da capacidade funcional aumenta a taxa de mortalidade dessa população, por prejudicar a autonomia do idoso. Dessa forma, o 


\section{Atenção à Saúde}

diagnóstico precoce e a prevenção são os maiores aliados para a manutenção da longevidade e independência do idoso ${ }^{9}$.

$O$ déficit cognitivo pode manifestar-se durante 0 processo de envelhecimento com início e avanços variável, relacionando-se com as próprias perdas biológicas inerentes ao tempo e à cultura do indivíduo. Além de que, os níveis sociais, econômicos, educacionais e a idade interferem no desempenho $\operatorname{cognitivo~}^{18}$.

Nesta pesquisa foi observado então, que os idosos que apresentaram menor nível cognitivo (09 participantes) estavam entre 65 a 80 anos. Sendo que apenas 01 entre eles com 60 anos apresentou maior nível cognitivo, estando este na média.

Em inúmeros casos, o déficit cognitivo pode relacionar-se à presença de incapacidade funcional no desempenho de atividades da vida diária, especialmente, em populações com baixo nível de escolaridade. Um estudo relacionado em uma unidade ambulatorial de atendimento ao idoso apresentou resultado que a associação com a prática de exercícios físicos e de lazer foi importante; sendo que os sedentários apresentaram prevalência de déficit cognitivo cerca de duas vezes maior do que os ativos ${ }^{19}$.

O envelhecimento cerebral também apresenta um desenvolvimento ímpar, pois quanto mais o cérebro for utilizado em atividades intelectuais, mais tempo ele demorará para perder suas conexões. Assim, pode apresentar uma perda sintomática, indicando sua excepcional capacidade plástica ${ }^{18}$.

A população idosa tem um alto índice de crescimento demográfico, e as instituições asilares constituem-se, muitas vezes, na única opção para esses indivíduos e suas famílias. Portanto, é essencial que essas instituições possuam uma boa estrutura, tanto em recursos humanos, quanto materiais para assim poder atender, convenientemente, a essa clientela. Com as especificidades relacionadas à faixa etária, o que pode indicar que $\mathrm{o}$ atendimento multidisciplinar baseado na prevenção primária representaria a manutenção da independência funcional e expectativa da vida com maior qualidade ${ }^{20}$.

Dessa forma, é necessário considerar que a pessoa idosa que está em uma instituição, pode possuir uma saúde mais vulnerável tanto nos aspectos físicos, como nos emocionais e sociais. E diante dessa fragilidade, nota-se que é preciso 
Artigo Original

Atenção à Saúde

amparar esses idosos com uma estrutura física e com atendimentos multidisciplinares adequados a demanda que esses indivíduos necessitam. Importante destacar que uma das limitações deste estudo foi o tamanho da amostra.

\section{CONCLUSÃO}

Este trabalho teve como objetivo geral avaliar depressão, ansiedade e nível cognitivo em idosos de uma instituição do município de Criciúma. Neste estudo, foi verificado que dos 15 idosos participantes, 07 apresentaram depressão, sendo que 04 entre os pesquisados são mulheres e 03 são homens.

Em relação a ansiedade, 04 idosos apresentaram indícios deste transtorno, sendo que 03 entre eles são homens. Entre os que indicaram ansiedade, 02 também apresentaram indícios de depressão, entre eles, 01 homem e 01 mulher.

Entre os 07 idosos que apresentaram indícios de depressão, 06 deles não fazem atividades físicas e nenhum participa de outras atividades sociais fora da instituição asilar. Os 04 idosos que apresentaram ansiedade tanto leve como moderada, 02 deles não praticam exercícios físicos e nenhum deles participa de atividades fora do asilo. Tendo como base outras publicações relacionando atividades psicossociais, depressão e ansiedade, há hipótese de que o declínio das atividades psicossociais pode estar relacionado ao desenvolvimento da depressão, assim como pode também desencadear ansiedade ${ }^{21,22}$.

Dentre os 07 participantes que apresentaram depressão, 05 apresentaram QI extremamente abaixo da média, entre estes, 03 homens e 02 mulheres. $\mathrm{E}$ os 04 idosos que apresentaram ansiedade, 03 entre eles também indicaram QI extremamente abaixo, sendo 01 mulher e 02 homens.

Foi observado nesta pesquisa que dos 09 idosos que apresentaram QI extremamente abaixo da média, 08 possuíam baixa escolaridade, ou seja, ensino fundamental incompleto. Em relação ao gênero, 06 são homens e 03 são mulheres.

Entre esses 08 idosos com QI extremamente abaixo e baixa escolaridade, 05 deles há indícios de depressão e 02 deles também apresentaram ansiedade leve e 01 ansiedade moderada. Um (01) participante com QI extremamente abaixo da média e baixa escolaridade apresentou somente ansiedade leve. 
Os participantes que apresentaram QI extremamente abaixo, baixa escolaridade, depressão e ansiedade em comorbidade foram 01 homem e 01 mulher. Somente 01 homem apresentou ansiedade, QI extremamente abaixo e baixa escolaridade e 02 homens e 01 mulher depressão, QI extremamente abaixo e baixa escolaridade. Há estudos que indicam que um dos fatores que se relaciona à quadros depressivos, assim como entre outras comorbidades, é a baixa escolaridade que o indivíduo apresenta ${ }^{23}$.

Uma das soluções para ajudar a suprir o cuidado com os idosos institucionalizados poderia ser o uso terapêutico baseado na iniciação de programas ocupacionais nas instituições asilares para assim poder gerar uma manutenção na qualidade de vida dos idosos e contribuir no dia a dia desses indivíduos ${ }^{24}$.

No estudo de Gregorutti e Araújo (2012), foi constatado que a relação do idoso institucionalizado com atividades expressivas gerou um progresso na expressão corporal, comunicação verbal, interação grupal, motivação e iniciativa ${ }^{25}$.

Esses resultados geram mais clareza para entender que a ocupação interfere positivamente no bem-estar nos idosos. Possivelmente atividades expressivas podem contribuir para a mudança de comportamento ocupacional no processo terapêutico com os idosos institucionalizados ${ }^{26}$.

A utilização de atividades expressivas é importante importante para a prática da terapia ocupacional, pois assim, os idosos que estão institucionalizados podem ocupar seu tempo diariamente com atividades que permitem-Ihes sentiremse úteis a cada dia.

Em relação ao tempo de institucionalização; entre os 09 idosos que apresentaram QI extremamente abaixo da média, 07 participantes estão há mais de 02 anos institucionalizados. Dentre os idosos que apresentaram depressão e ansiedade, 06 deles estão há mais de 02 anos no asilo.

A pessoa idosa que está institucionalizada pode apresentar determinadas fragilidades, pois seu aspecto tanto físico como social e emocional estará afetado já que a instituição asilar resulta em um afastamento social, primeiramente, pois quem está institucionalizado consequentemente não tem muita proximidade de seus familiares e conhecidos. Considerando também o novo ambiente em que o idoso terá que se adaptar, no caso o asilo. 
Artigo Original

Atenção à Saúde

Através dos resultados sugere-se ter relação a comorbidade entre depressão e ansiedade, além de baixa escolaridade. Diante desse contexto, sugere-se para as próximas pesquisas um estudo que relacione ansiedade, depressão e comprometimento cognitivo (QI) com o tempo de permanência dos idosos em instituições asilares.

\section{REFERÊNCIAS}

1. Organización Panamericana de la Salud (OPAS). Enfermaria gerontologica: conceptos para la práctica. Washington. Elías Anzola Pérez. Série PALPEX, n.31. 1993.

2. Cordeiro FR, Pinheiro MS, Correio DAC. Sobre o envelhecimento, a mídia e a morte no contemporâneo. Estud. Interdiscipl. Envelhec. 2015;975-90.

3. Brasil. Ministério da Saúde. Secretaria de Atenção à Saúde. Departamento de Atenção Básica. Envelhecimento e saúde da pessoa idosa. MS. 2006.

4. Guimarães $A$, et al. Ansiedade e parâmetros funcionais respiratórios de idosos praticantes de dança. Fisioter. Mov. 2011;24(4):683-8.

5. Marconi, Marina de Andrade; Lakatos, Eva Maria. Fundamentos de metodologia científica. 7ª ed. São Paulo: Atlas, 2010.

6. Wechsler, David. WASI - Escala Wechsler Abreviada de Inteligência. $1^{\underline{a}}$ ed. Casa do Psicólogo, 2014.

7. Beck AT, Brown G, Epstein N, Steer RA. Inventário para medir ansiedade clínica. Jornal de consultoria e psicologia clínica. 1988;56:893-7.

8. Souza $R L$, et al. Validade e fidedignidade da escala de depressão geriátrica na identificação de idosos deprimidos em um hospital geral. J Bras Psiquiatr. 2007;56(2):102-7.

9. Trindade APNT, et al. Repercussão do declínio cognitivo na capacidade funcional em idosos institucionalizados e não institucionalizados. Fisioter. Mov. 2013;26(2):281-9.

10. Oliveira $D$, et al. Prevalência de depressão em idosos que frequentam centros de convivência. Rev. Saúde Pública. 2006;40(4):734-6.

11. Byers AL, et al. High Occurrence of Mood and Anxiety Disorders Among Older Adults. American Medical Association. Arch Gen Psychiatry. 2010;67(5):489-96. 
12. Bottino $\mathrm{CMC}$, et al. Diagnóstico diferencial entre demência e transtornos psiquiátricos: Critérios diagnósticos e exames complementares. Dementia e Neuropsychologia 2011;5(suppl. 1):91-8.

13. Minguelli $B$, et al. Comparação dos níveis de ansiedade e depressão entre idosos ativos e sedentários. Rev. Psiq. Clín. 2013;40(2):71-6.

14. Diniz BSO, et al. Nível educacional e idade no desempenho no Miniexame do Estado Mental em idosos residentes na comunidade. Rev. Psíq. Clín. 2007;34(1):137.

15. Abreu ID, et al. Demência de Alzheimer: correlação entre memória e autonomia. Rev. Psiq. Clín. 2005;32(3):131-6.

16. Carvalho M, et al. Quedas e fatores associados em idosos institucionalizados no município de Pelotas (RS, Brasil). Ciência e saúde coletiva. 2011;16(6):2945-52.

17. Dias DS, et al,. Comparação da percepção subjetiva de qualidade de vida e bem-estar de idosos que vivem sozinhos, com a família e institucionalizados. Rev. Bras. Geriatr. Gerontol. 2013;16(1):127-38.

18. NORDON, David Gonçalves et al., Perda cognitiva em idosos. Rev. Fac. Ciênc. Méd. 2009:11(3):5-8.

19. Cavalini LT, Chor D. Inquérito sobre hipertensão arterial e deficit cognitivo em idosos de um serviço de geriatria. Rev. Bras. Epidemiol. 2003;6(1):7-17.

20. Yamamoto A, Diogo MJD. Os idosos e as intituições asilares do município de ampinas. Rev Latino-am Enfermagem. 2002;10(5):660-6.

21. Djernes JK. Prevalence and predictors of depression in populations of elderly: a review. Acta Psychiatr Scand. 2006;113:372-87.

22. CHEIK, Nadia Carla et al., Efeitos do exercício físico e da atividade física na depressão e ansiedade em indivíduos idosos. R. Bras. Ci. E Mov. Brasília. 2003;11(3):45-52.

23. Pinho $M X$, Custório $O$, Makdisse $M$. Incidência de depressão e fatores associados em idosos residentes na comunidade: revisão de literatura. Rev. Bras. Geriatr. Gerontol. 2009;12(1):123-40.

24. Soares E. Projeto Memória e Envelhecimento: capacitando profissionais e aprimorando aspectos cognitivos em idosos institucionalizados. Revista Brasileira de Ciências do Envelhecimento Humano. 2010;7(1):62-73.

25. Gregorutti CC, Araújo RCT. Idosos institucionalizados e depressão: atividades expressivas e seu potencial terapêutico. Revista Brasileira de Ciências do Envelhecimento Humano. 2012;9(2):274-81. 
Artigo Original

Atenção à Saúde

26. Clarkson-Smith L, Hartlhey AA. The game of brige as na exercise in working memory and reasoning. Journal of Gerontology. 1990:45(6):233-8. 